\title{
Factors That Influence Treatment Completion for Latent Tuberculosis Infection
}

\author{
McKenna C. Eastment, MD, MPH, Adelaide H. McClintock, MD, \\ Christy M. McKinney, PhD, MPH, Masabiro Narita, MD, and Alexandra Molnar, MD
}

Introduction: The aim of this study is to describe factors associated with noncompletion of latent tuberculosis infection (LTBI) therapy.

Methods: We conducted a retrospective cohort study of adults who initiated LTBI treatment with isoniazid, rifampin, or isoniazid-rifapentine at 5 clinics. Demographic, treatment, and monitoring characteristics were abstracted. We estimated descriptive statistics and compared differences between completers and noncompleters using $t$ tests and $\chi^{2}$ tests.

Results: The rate of completion across LTBI regimens was $66 \%(n=393)$. A greater proportion of noncompleters were unmarried, used tobacco and/or alcohol, and had more medical problems than completers (all $P<.05)$. A larger proportion of noncompleters received charity care compared with completers $(P<.001)$. The most common reason for treatment discontinuation was loss to follow-up; the majority of these participants were treated with the longest isoniazid-only regimen.

Conclusions: Patients at risk of progression to active tuberculosis with factors associated with noncompletion may benefit from interventions that enhance adherence to LTBI therapy. These interventions could include enhanced outreach, incentive programs, or home visits. ( $\mathrm{J}$ Am Board Fam Med 2017;30: 520-527.)

Keywords: Antitubercular Agents, Isoniazid, Latent Tuberculosis, Motivation, Retrospective Studies, Rifampin, Rifapentine

An estimated 11 million people have latent tuberculosis infection (LTBI) in the United States. ${ }^{1}$ Despite studies demonstrating prevention of progression to active tuberculosis (TB), ${ }^{2-4}$ cost savings, ${ }^{5,6}$ and quality-adjusted life years saved with treat-

This article was externally peer reviewed.

Submitted 23 February 2017; revised 15 March 2017; accepted 22 March 2017.

From the Division of Allergy and Infectious Diseases, Department of Medicine, University of Washington School of Medicine, Seattle (ME); the Division of General Internal Medicine, Department of Medicine, University of Washington School of Medicine, Seattle (AHM, AM); the Department of Oral Health Sciences, University of Washington School of Dentistry, Seattle (CMM); the Division of Pulmonary \& Critical Care Medicine, Department of Medicine, University of Washington School of Medicine, Seattle (MN); and the Tuberculosis Control Program, Public Health - Seattle \& King County, Seattle, Washington (MN).

Funding: This study was supported by the Division of General Internal Medicine; data support was received from the Institute of Translational Health Sciences (UL1 RR025014 from the National Center for Research Resources/National Institutes of Health), both to the University of Washington. MCE was funded by the National Institutes of Health STD \& AIDS Research Training Program (T32 AI07140). ment of LTBI, ${ }^{5}$ the proportion of patients who complete LTBI treatment remains small. Many of the treatment challenges stem from low adherence in "actual use" scenarios in the community, often with patients experiencing many medical problems, potential substance use issues, and social upheaval. The standard regimen, isoniazid daily for 9 months, has a completion rate $<60 \%{ }^{7-9}$

Currently, the Centers for Disease Control and Prevention recommends 3 regimens: daily isoniazid for 9 months, ${ }^{10}$ daily rifampin for 4 months, ${ }^{10}$ or weekly isoniazid and rifapentine with directly observed therapy (DOT) for 3 months. ${ }^{10,11}$ While a shorter course of treatment improves completion rates, ${ }^{12}$ understanding which patients may be at risk for noncompletion can help providers identify pa-

Conflict of interest: none declared.

Corresponding author: McKenna C. Eastment, MD, MPH, 1959 NE Pacific Street, Box 356423, Seattle, WA, 98195 (E-mail: mceast@uw.edu). 
tients, especially those at risk of progression to active tuberculosis (TB), who may need enhanced support or case management to ensure completion.

Prior research identified factors associated with noncompliance related to choice of regimen. Both the duration of the treatment regimen ${ }^{8,13,14}$-with shorter regimens achieving better rates of completion-and side effects ${ }^{15}$ are associated with noncompletion of LTBI therapy. Further research efforts have focused on trying to identify patientlevel factors associated with noncompletion. Being married seems to be a positive predictor of completion. ${ }^{13}$ Several studies demonstrated patient transportation or clinic access issues as a barrier to completion. ${ }^{16-20}$ This is particularly important to consider with the newer DOT therapies available. Homelessness, ${ }^{13,20}$ alcohol use, ${ }^{13}$ and intravenous drug use ${ }^{21}$ also all seem to be associated with noncompletion. While some studies demonstrate an impact of age on completion, with younger patients less likely to complete therapy, ${ }^{13}$ others failed to replicate this finding. ${ }^{22}$ Similarly mixed findings exist for variables such as level of education, ${ }^{22}$ number of years spent living in the United States, ${ }^{22}$ and being foreign born. ${ }^{13}$ One study demonstrated that men are more likely to complete therapy than women. ${ }^{20}$ However, most of these studies were completed before the approval and wide use of the 3 -month isoniazid and rifapentine regimen and before the increasing use of the 4-month daily rifampin regimen, and thus do not provide information on the challenges of additional treatment options.

This study aims to add to the literature about factors for noncompletion related to these newer, shorter LTBI treatment regimens. The purpose of this study is to describe factors associated with noncompletion of therapy for 3 LTBI regimens. Insights from this study can be used to identify modifiable factors and design interventions to improve adherence.

\section{Methods}

We conducted a retrospective cohort study of patients who initiated treatment for LTBI with 9 months of isoniazid, 4 months of rifampin, or 3 months of isoniazid and rifapentine. We enrolled patients $\geq 18$ years old who were HIV negative and not pregnant. HIV-positive patients were excluded because rifamycin-based regimens have potential drug-drug interactions with many antiretrovirals that this population may be taking. Patients who were treated at 1 of 4 different hospital-affiliated clinics associated with an academic medical center in Seattle, Washington, received their medications from a hospital-based pharmacy. The 4 clinics included a primary care clinic for homeless patients, an employee health clinic, an infectious diseases clinic, and an international/refugee clinic. A fifth site was a county TB clinic.

Data were extracted by 3 of the authors (ME, AHM, AM) from the electronic medical record and paper charts (county TB clinic) and data were entered using a REDCap project database. ${ }^{23} \mathrm{We}$ abstracted age, sex, marital status, and insurance status from the electronic medical record. Charity care is defined as those who met poverty guidelines but could not afford insurance and were provided free care through the medical center. If information on marital or insurance status was unable to be obtained from the chart or was unclear, it was marked as unknown. Patients were classified as using alcohol only if alcohol abuse or dependence was listed in a problem list, or the social history documentation noted that they drink more than 3 alcohol-containing drinks per day. Otherwise, the person was classified as not being an alcohol user. Homelessness was defined as living on the street, in shelters, abandoned buildings, cars, or tents, or as staying in housing the patient identified as temporary, which is a more inclusive definition than the federal definition of homelessness. ${ }^{24}$ Per a review of social documentation, those who met this definition were classified as homeless; all others were classified as not homeless. During the second half of the study period, a special incentive program was in place for homeless patients: those seen at the TB clinic and the homeless clinic were given special incentives, including bus fare or a $\$ 10$ meal voucher so that medicine could be taken with food. Patients at the TB clinic were provided medications free of charge, as they were either recent contacts of an infectious TB case or high-risk immigrants/refugees. Side effects such as nausea, vomiting, abdominal pain, neuropathic pain, rash, liver function test (LFT) abnormalities, or other laboratory abnormalities were evaluated. Side effects were classified as "other" if the patient reported a subjective side effect that was not a known side effect of the medication regimens.

Among patients who did not complete therapy, the reasons for discontinuation included loss to 
follow-up, side effects, "other" reasons, patient or provider concern, LFT abnormalities, finances, or other laboratory abnormalities. Pharmacy records were used to define treatment completion. Patients were deemed to have completed therapy if they filled consecutive prescriptions that corresponded to 270 doses of isoniazid within 12 months, ${ }^{10} 120$ doses of rifampin within 6 months, ${ }^{10}$ and 12 doses of isoniazid and rifapentine within 4 months. ${ }^{11} \mathrm{~A}$ large proportion of the data abstraction was done by consensus among coauthors. In addition, $10 \%$ of charts were randomly selected and reviewed by the coauthors for accuracy.

We estimated the proportion who completed treatment across all regimens combined. We calculated counts, proportions, and means for demographic, treatment, and health characteristics for all participants combined and by treatment completion status. We used $\chi^{2}$ tests to compare completers with noncompleters on demographic, social, and behavioral factors. To better understand adverse effects of treatment, we calculated the distribution of side effects across type of treatment among those reporting side effects. Among those who discontinued treatment, we estimated the proportion reporting various reasons for noncompletion across type of treatment. All analyses were conducted using Stata 13.1 (Stata Corp, College Station, TX).The Human Subjects Division of the University of Washington reviewed and approved this research.

\section{Results}

A total of 393 participants were included in this analysis. A total of 259 participants (66\%) completed therapy (completers), whereas 132 (34\%) did not complete therapy (noncompleters) (Table 1); data were missing for 2 participants. A total of 87 patients were treated with isoniazid and rifapentine, 82 with rifampin alone, and 224 with isoniazid alone. Participants treated with the isoniazid and rifapentine regimen had completion rates similar to those treated with rifampin alone, which was statistically higher than those treated with isoniazid alone (Table 1). These associations remained similar in direction and statistically significant after adjusting for clinic location and type of monitoring (data not shown).

Almost half of patients who completed (51.0\%) and did not complete (47.8\%) therapy received charity care as coverage for their medical care (Ta- ble 1). Among patients who completed therapy, $91 \%$ were foreign born, which was similar to the $90.2 \%$ of noncompleters who were foreign-born. Among those with data on marital status $(60 \%)$, almost two thirds of noncompleters were married $(60.8 \%)$ and $73.7 \%$ of completers were married. A minority of patients were homeless $(15.0 \%$ of the noncompleters and $10.7 \%$ of the completers).

A larger proportion of noncompleters were unmarried, used tobacco and/or alcohol, and had more medical problems compared with completers (all $P<.05$ ) (Table 1). A larger proportion of noncompleters received charity care compared with completers $(P<.001)$. The proportions of completers and noncompleters did not differ by those who spoke English, were homeless, were experiencing side effects, or were taking more medications (Table 1). The number of patients using intravenous drugs $(n=2)$ was too small for statistical analyses.

Loss to follow-up was the most common reason for noncompletion (Figure 1), and other laboratory abnormalities was the least common reason. Those patients receiving the longest regimen (isoniazid alone) were most often lost to follow-up (53 of the 64 total participants who were lost to follow-up). Only patients in the isoniazid and rifapentine treatment group reported finances as a reason for noncompletion. LFT abnormalities were a cause of discontinuation in the isoniazid-only group and, to a lesser extent, in the combination isoniazid and rifapentine group. LFT abnormalities were not a cause of discontinuation for anyone in the rifampin-only group (Figure 1).

Approximately one third of all patients reported at least 1 side effect (Figure 2). Small numbers of patients did report nausea, vomiting, abdominal pain, neuropathic pain, or rash, but the most commonly reported side effects were categorized as "other" (ie, they did not fall under the previously defined categories) and were not typically associated with the study medications (eg, facial pain) (Figure 2).

\section{Discussion}

This article characterizes factors associated with completion and noncompletion of the 3 most common regimens for LTBI treatment at 5 academic clinics. Notably, a lack of insurance was associated with less treatment completion, and being married 


\begin{tabular}{|c|c|c|c|}
\hline & $\begin{array}{l}\text { Noncompleters } \\
\quad(\mathrm{n}=132)\end{array}$ & $\begin{array}{l}\text { Completers } \\
(\mathrm{n}=259)\end{array}$ & $P$ Value \\
\hline Age (years), mean & 41.6 & 44.6 & .06 \\
\hline \multicolumn{4}{|l|}{ Sex } \\
\hline Female & $53(40.2)$ & $122(47.1)$ & \multirow[t]{2}{*}{.19} \\
\hline Male & $79(59.9)$ & $137(52.9)$ & \\
\hline \multicolumn{4}{|l|}{ Treatment regimen } \\
\hline Isoniazid and rifapentine & $13(9.9)$ & $74(28.6)$ & \multirow[t]{3}{*}{$<.001$} \\
\hline Rifampin alone & $12(9.1)$ & $70(27.0)$ & \\
\hline Isoniazid alone & $107(81.1)$ & $115(44.4)$ & \\
\hline \multicolumn{4}{|l|}{ Type of insurance* } \\
\hline Private & $10(8.7)$ & $49(23.3)$ & \multirow[t]{4}{*}{$<.001$} \\
\hline Medicare/Medicaid/funded by government & $30(26.1)$ & $45(21.4)$ & \\
\hline Charity/no insurance & $55(47.8)$ & $107(51.0)$ & \\
\hline Other & $20(17.4)$ & $9(4.3)$ & \\
\hline \multicolumn{4}{|l|}{ Race } \\
\hline White & $11(9.1)$ & $18(7.1)$ & \multirow[t]{6}{*}{.10} \\
\hline Black & $39(32.2)$ & $55(21.6)$ & \\
\hline Asian & $46(38.0)$ & $115(45.1)$ & \\
\hline Mexican/other Hispanic & $16(13.2)$ & $32(12.6)$ & \\
\hline Pacific Islander & $5(4.1)$ & $27(10.6)$ & \\
\hline Other & $4(3.3)$ & $8(3.1)$ & \\
\hline Foreign born & $110(90.2)$ & $230(91.3)$ & .73 \\
\hline English speaking & $66(50.8)$ & $136(53.3)$ & .63 \\
\hline \multicolumn{4}{|l|}{ Marital status $^{\dagger}$} \\
\hline Married & $48(60.8)$ & $115(73.7)$ & \multirow[t]{2}{*}{.04} \\
\hline Unmarried/separated & $31(39.2)$ & $41(26.3)$ & \\
\hline Homeless $^{\ddagger}$ & $18(15.0)$ & $26(10.7)$ & .24 \\
\hline Experienced side effects & $37(28.0)$ & $94(36.3)$ & .10 \\
\hline \multicolumn{4}{|l|}{ Medical problems (n) } \\
\hline 0 & $47(35.6)$ & $130(50.2)$ & \multirow[t]{3}{*}{.01} \\
\hline $1-2$ & $42(31.8)$ & $77(29.7)$ & \\
\hline$\geq 3$ & $43(32.6)$ & $52(20.1)$ & \\
\hline \multicolumn{4}{|l|}{ Medications (n) } \\
\hline $0-1$ & $45(34.1)$ & $93(35.9)$ & \multirow[t]{3}{*}{.86} \\
\hline $2-3$ & $49(37.1)$ & $89(34.4)$ & \\
\hline$\geq 3$ & $38(28.8)$ & $77(29.7)$ & \\
\hline Tobacco use & $23(17.4)$ & $26(10.0)$ & .04 \\
\hline Alcohol use ( $>3$ drinks, abuse, or dependence) & $25(18.9)$ & $27(10.4)$ & .02 \\
\hline
\end{tabular}

Data are $\mathrm{n}(\%)$ unless otherwise indicated. Also unless indicated, $<5 \%$ of data was missing.

${ }^{*}$ Missing data: $16.8 \%$.

${ }^{\dagger}$ Missing data: $40.0 \%$.

${ }^{\ddagger}$ Missing data: $7.1 \%$.

and abstaining from tobacco and alcohol use were associated with successfully completing treatment. Based on prior studies, the type and duration of treatment are likely 2 of the most significant factors in predicting treatment completion for latent tuberculosis. ${ }^{9,14}$ In a separate study, we found that the use of DOT was not associated with treatment completion when adjusted for type of treatment. ${ }^{14}$
However, many additional factors likely predict treatment completion, such as a patient's understanding of and motivation to take LTBI treatment, interaction between the patient and the prescribing medical provider and clinical staff, the perception and true incidence of side effects, and the response to these events by the patient and the medical provider. 
Figure 1. These are the numbers of patients with specific reasons for non-completion by treatment regimen. Being lost to follow-up was the most common reason for treatment non-completion. The most patients were lost to follow-up if they were treated with the longest regimen of isoniazid only.

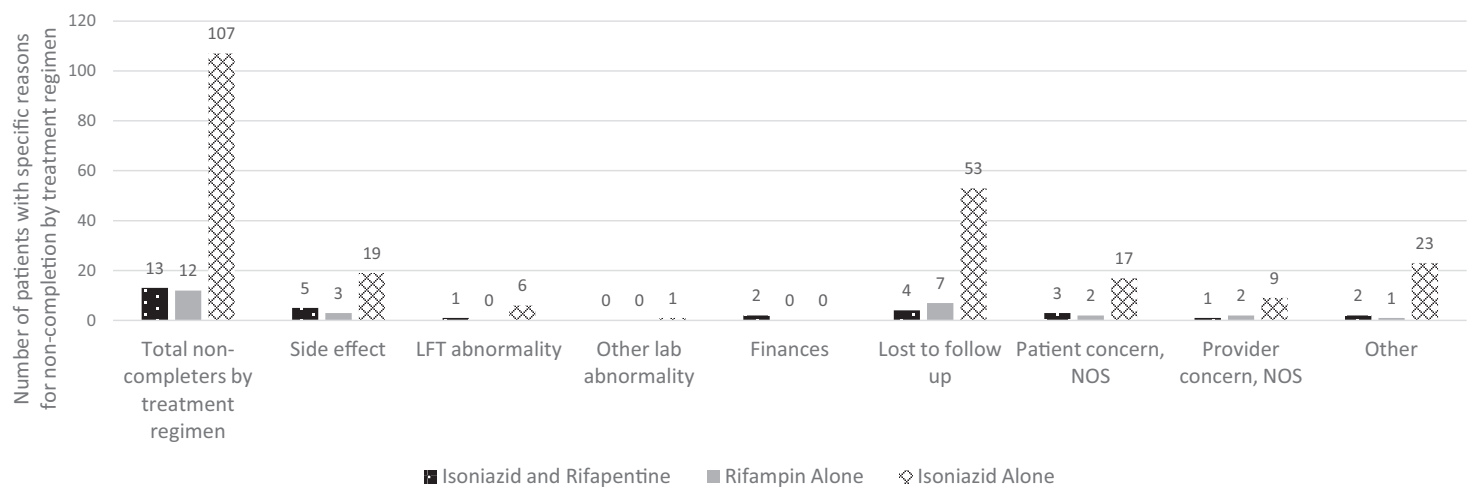

Our study demonstrates that people receiving charity care are less likely than those with private insurance to complete treatment, which is consistent with the findings of other studies. ${ }^{20,25}$ Among the subjects who initiated treatment in 2013 or later, the Affordable Care Act had made insurance more broadly available to all but undocumented immigrants. Washington State, where this study was done, expanded Medicaid, and so by 2013 the majority of patients were insured. It is notable that this difference persists even though people without insurance or with charity care receive their LTBI treatment free at all the clinic sites in our study. Lack of resources for even basic insurance is likely a marker for poverty or possibly undocumented status, which may mean that patients had fewer resources and/or support to come to the clinic and pick up their medicines, even though these medi- cines and clinic visits were free. These individuals would likely benefit most from early initiation of enhanced adherence models and support for completion that may include more intensive outreach or home visits. In addition, training medical providers who care for patients with LTBI may improve patients' engagement in treatment and provide skills to handle minor side effects without discontinuing medication, particularly for patients at risk for progression to active TB. Last, cost was listed as a reason for treatment noncompletion only by those patients taking isoniazid and rifapentine. In general, medications were free or insurance covered the medications, and so it is possible that the barrier to this regimen is the cost associated with participating in DOT. Providers also need to take into consideration patients' ability to participate in DOT, as this may be a major barrier to completion.

Figure 2. These are the numbers of participants experiencing specific side effects by treatment regimen. Approximately one-third of patients reported at least one side effect.

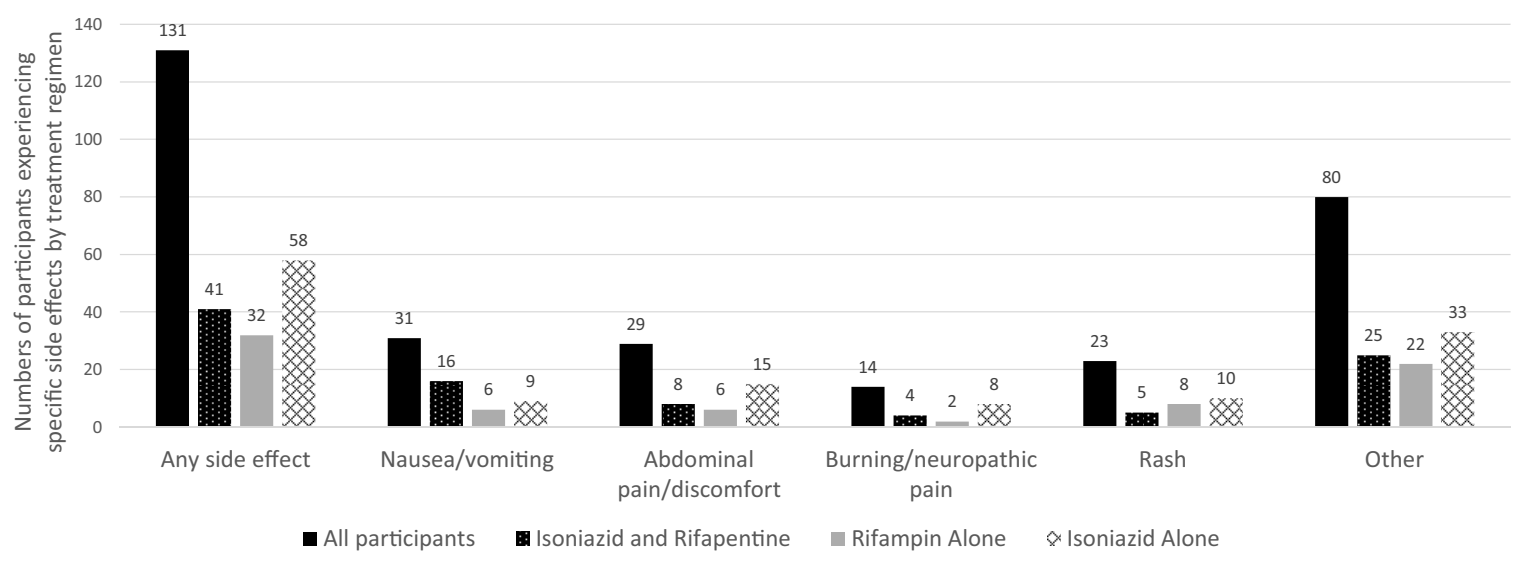


Consistent with other studies, ${ }^{13,21}$ we found that individuals who were using alcohol or tobacco at the start of therapy completed therapy statistically significantly less often than those not misusing alcohol or tobacco at the start of therapy. We did not find a statistically significant association between drug use and treatment failure, but the numbers for drug use in this study were quite small. This is likely because most providers at the clinic sites do not initiate LTBI treatment for individuals with active intravenous drug use because of historically poor adherence.

A number of studies have found positive social networks and supportive relationships to enhance health and adherence to medications. ${ }^{13,21} \mathrm{We}$ also found this to be the case with married patients. This may aid in alerting prescribing providers to who may be more likely to complete therapy and who may benefit from enhanced adherence support.

An increased number of medical problems was associated with noncompletion. A high number of medical and/or social problems may delay or prevent initiation of treatment for LTBI in the first place, because so many other more pressing issues need to be covered during a brief clinic visit. The number of medications was not associated with treatment failure, suggesting that people taking multiple medications may develop mechanisms for remembering their medicines that make up for the complexity. Poor adherence associated with medical complexity has been poorly described to date in the LTBI treatment adherence literature and is an important factor when deciding who merits enhanced adherence practices.

We found that side effects suggest increasing rates of noncompletion, though this was not statistically significant. Other studies reported higher rates of treatment failure associated with side effects. ${ }^{8,25}$ This could be a limitation of our sample size or a result of comparing the 3 LTBI regimens, 1 of which is relatively new. This does highlight that providers may want to consider more than just the side effect profile when deciding on an LTBI regimen for patients. Given our results about who completes and who does not complete therapy, providers may want to also consider the duration of the regimen and the need for DOT.

We were surprised to find that country of birth, English proficiency, and homelessness did not affect completion rates, as has been noted in other studies. ${ }^{13}$ At our clinic sites that primarily serve homeless and foreign-born populations, preexisting models are in place to enhance adherence, such as incentive programs ${ }^{26}$ and cultural mediators/case managers for certain groups of newly arrived refugees. ${ }^{27}$ These cultural mediators/case managers aim to enhance health literacy in a culturally appropriate way for patients new to the United States, who may not be familiar with medical/clinic practices. This could explain the lack of a difference in completion rates for these populations.

Being "lost to follow-up" was a frequent reason for failure to complete therapy, and this has also been demonstrated in another study. ${ }^{8}$ The most participants were lost to follow-up in the isoniazidonly regimen, which is the longest regimen of the 3 included in this analysis. This finding argues for careful screening and education before initiating therapy and during treatment in order to enhance the likelihood of follow-up. The reasons for being lost to follow-up are unknown and should be investigated in future studies.

This study is helpful in characterizing factors associated with noncompletion of all 3 of the most common LTBI treatment regimens in the United States, but it has several limitations. This study included 5 clinics in 1 city, which could affect the generalizability of these findings to other populations. Retrospective chart review has inherent limitations due to the inability to capture all information, and information is occasionally missing or poorly documented in charts, which may limit the accuracy of our findings. Side effects were identified by patient report, which could lead to under-reporting if patients are lost to follow-up. Our method of determining completion based on pharmacy records did not include pill counts or other methods to directly test adherence. Last, this was a convenience sample of patients being treated for LTBI, and not randomly selected patients; therefore the sample may not be representative of the entire population.

\section{Conclusions}

Overall, we found that a lack of insurance is significantly associated with lower likelihood of treatment completion, despite free medicines and no-cost visits. We also found that marital status and abstinence from tobacco and alcohol are more closely associated with successfully completing treatment. We hope this informs practitioners about which patients may have barriers to adherence and who may benefit from en- 
hanced adherence efforts such as enhanced outreach, incentive programs, home visits, and those described in other studies. ${ }^{21,26,28,29}$

The authors acknowledge Caroline L. Pitney, PharmD, for in-depth data mining in the early stages of this project; David R. Park, MD, Shireesha Dhanireddy, MD, and John Lynch, MD, $\mathrm{MPH}$, for participating in the formulation of this project; and Monica Pecha, MPH, and the public health TB clinic staff for their kind assistance with data sets and charts at the Tuberculosis Control Program, Public Health - Seattle \& King County.

To see this article online, please go to: http://jabfm.org/content/ 30/4/520.full.

\section{References}

1. Bennett DE, Courval JM, Onorato I, et al. Prevalence of tuberculosis infection in the United States population: the national health and nutrition examination survey, 1999-2000. Am J Respir Crit Care Med 2008;177:348-55.

2. Ferebee, SH. Controlled chemoprophylaxis trials in tuberculosis. A general review. Bibl Tuberc 1970;26: 28-106.

3. Smieja MJ, Marchetti CA, Cook DJ, Smaill FM. Isoniazid for preventing tuberculosis in non-HIV infected persons. Cochrane Database Syst Rev 2000; (2):CD001363.

4. Churchyard GJ, Fielding KL, Lewis JJ, et al. A trial of mass isoniazid preventive therapy for tuberculosis control. N Engl J Med 2014;370:301-10.

5. Porco TC, Lewis B, Marseille E, Grinsdale J, Flood JM, Royce SE. Cost-effectiveness of tuberculosis evaluation and treatment of newly-arrived immigrants. BMC Public Health 2006;6:157.

6. Holland DP, Sanders GD, Hamilton CD, Stout JE. Costs and cost-effectiveness of four treatment regimens for latent tuberculosis infection. Am J Respir Crit Care Med 2009;179:1055-60.

7. Goswami ND, Gadkowski LB, Piedrahita C, et al. Predictors of latent tuberculosis treatment initiation and completion at a U.S. public health clinic: a prospective cohort study. BMC Public Health 2012;12: 468.

8. Hess K, Goad J, Wu J, Johnson K. Isoniazid completion rates for latent tuberculosis infection among college students managed by a community pharmacist. J Am Coll Health 2009;57:553-5.

9. Rivest P, Street MC, Allard R. Completion rates of treatment for latent tuberculosis infection in Quebec, Canada from 2006 to 2010. Can J Public Health 2013;104:e235-9.

10. Targeted tuberculin testing and treatment of latent tuberculosis infection. American Thoracic Society. MMWR Recomm Rep 2000;49(RR-6):1-51.

11. Centers for Disease Control and Prevention (CDC). Recommendations for use of an isoniazid-rifapentine regimen with direct observation to treat latent $\mathrm{My}-$ cobacterium tuberculosis infection. MMWR Morb Mortal Wkly Rep 2011;60:1650-3.

12. Sharma SK, Sharma A, Kadhiravan T, Tharyan P. Rifamycins (rifampicin, rifabutin and rifapentine) compared to isoniazid for preventing tuberculosis in $\mathrm{HIV}$-negative people at risk of active TB. Cochrane Database Syst Rev 2013;(7):CD007545.

13. Hirsch-Moverman Y, Bethel J, Colson PW, Franks J, El-Sadr W. Predictors of latent tuberculosis infection treatment completion in the United States: an inner city experience. Int J Tuberc Lung Dis 2010; 14:1104-11.

14. McClintock AH, Eastment M, McKinney CM, et al. Treatment completion for latent tuberculosis infection: a retrospective cohort study comparing 9 months of isoniazid, 4 months of rifampin and 3 months of isoniazid and rifapentine. BMC Infect Dis 2017;17:146.

15. Machado A Jr, Finkmoore B, Emodi K, et al. Risk factors for failure to complete a course of latent tuberculosis infection treatment in Salvador. Brazil. Int J Tuberc Lung Dis 2009;13:719-25.

16. Shieh FK, Snyder G, Horsburgh CR, Bernardo J, Murphy C, Saukkonen JJ. Predicting non-completion of treatment for latent tuberculous infection: a prospective survey. Am J Respir Crit Care Med 2006; 174:717-21.

17. Wyss LL, Alderman MK. Using theory to interpret beliefs in migrants diagnosed with latent TB. Online J Issues Nurs 2007;12:7.

18. West EL, Gadkowski LB, Ostbye T, Piedrahita C, Stout JE. Tuberculosis knowledge, attitudes, and beliefs among North Carolinians at increased risk of infection. N C Med J 2008;69:14-20.

19. McEwen MM, Boyle J. Resistance, health, and latent tuberculosis infection: Mexican immigrants at the U. S.-Mexico border. Res Theory Nurs Pract 2007; 21:185-97.

20. Hirsch-Moverman Y, Shrestha-Kuwahara R, Bethel $\mathrm{J}$, et al. Latent tuberculous infection in the United States and Canada: who completes treatment and why?. Int J Tuberc Lung Dis 2015;19:31-8.

21. Nyamathi AM, Christiani A, Nahid P, Gregerson P, Leake B. A randomized controlled trial of two treatment programs for homeless adults with latent tuberculosis infection. Int J Tuberc Lung Dis 2006;10: 775-82.

22. Ailinger RL, Black P, Nguyen N, Lasus H. Predictors of adherence to latent tuberculosis infection therapy in Latino immigrants. J Community Health Nurs 2007;24:191-8.

23. Harris PA, Taylor R, Thielke R, Payne J, Gonzalez N, Conde JG. Research electronic data capture (REDCap) - a metadata-driven methodology and workflow process for providing translational research informatics support. J Biomed Inform 2009; 42: $377-81$. 
24. US Code, Title 42, The Public Health and Welfare, 2000, 254b.

25. Kwara A, Herold JS, Machan JT, Carter EJ. Factors associated with failure to complete isoniazid treatment for latent tuberculosis infection in Rhode Island. Chest 2008;133: 862-8.

26. Gupta V, Sugg N, Butners M, Allen-White G, Molner A. Tuberculosis among the homeless-preventing another outbreak through community action. N Engl J Med 2015;372:1483-5.

27. Jackson-Carroll L, Graham E, Jackson JC. Beyond medical interpretation: the role of interpreter cultural mediators (ICMs) in building bridges between ethnic communities and health institutions. Select- ing, training and supporting key outreach staff. EthnoMed [Internet]Seattle: Harborview Medical Center; 1995 (revised 1998). Available from: http:// ethnomed.org/about/related-programs/communityhouse-calls-program/icm-manual98.pdf.

28. Ailinger RL, Martyn D, Lasus H, Lima Garcia N. The effect of a cultural intervention on adherence to latent tuberculosis infection therapy in Latino immigrants. Public Health Nurs 2010;27:115-20.

29. Chang AH, Polesky A, Bhatia G. House calls by community health workers and public health nurses to improve adherence to isoniazid monotherapy for latent tuberculosis infection: a retrospective study. BMC Public Health 2013;13:894. 